\title{
Similarity in joint function limitation in Type 3 von Willebrand's disease and moderate haemophilia A
}

\author{
S. L. SOOD,$*$ A. CUKER, $\dagger$ C. WANG, $*$ A. D. METJIAN, $\mathbb{S}$ E. Y. CHIANG, $\dagger$ J. M. SOUCIE, \\ B. A. KONKLE $₫$ and THE HTCN STUDY INVESTIGATORS ${ }^{1}$ \\ *Hemophilia and Coagulation Disorders Program, Division of Hematology-Oncology, University of Michigan School of \\ Medicine, Ann Arbor, MI; †Penn Comprehensive Hemophilia and Thrombosis Program, Division of Hematology-Oncology, \\ University of Pennsylvania, Philadelphia, PA; \$Centers for Disease Control (CDC), Atlanta, GA; \$Division of Hematology, Duke \\ University School of Medicine, Durham, NC; and $₫$ Puget Sound Blood Center and University of Washington, Seattle, WA
}

\begin{abstract}
Summary. Type 3 von Willebrand's disease (VWD) is a rare bleeding diathesis with complete or near complete deficiency of von Willebrand factor (VWF) and low factor VIII (FVIII) levels. In contrast, only FVIII is decreased in haemophilia A (HA). Both disorders are complicated by arthropathy. The purpose of this study was to further clarify the roles of FVIII and VWF: Antigen (VWF:Ag) in joint range of motion (ROM) loss over time. We compared joint ROM loss and other bleeding manifestations in 100 Type 3 VWD subjects $(\mathrm{FVIII} \leq 5 \%)$ and 1814 moderate HA subjects (FVIII 1$5 \%$ ) within the U.S. Universal Data Collection (UDC) database. High rates of bleeding were reported at baseline. During follow-up, moderate HA patients reported a joint $(46 \%$ vs. $34 \%, P<0.0001)$ or muscle bleed $(27 \%$ vs. $16 \%, P<0.0001)$ in a higher
\end{abstract}

proportion of visits than VWD patients. Other bleeds, including mucosal, were reported in a greater proportion of visits among patients with Type 3 VWD than among those with HA $(49 \%$ vs. $32 \%$, $P<0.0001)$. Multivariate analysis revealed no difference in joint ROM loss over time in the Type 3 VWD vs. moderate HA populations. A higher FVIII level was protective in both VWD and HA $(P<0.001)$. Our findings support the hypothesis of primacy of the FVIII level in determining risk of joint haemorrhage, and may help target therapy in Type 3 VWD and moderate HA to prevent joint disability.

Keywords: arthropathy, factor VIII, joint range of motion, moderate haemophilia A, von Willebrand factor, von Willebrand's disease

\section{Introduction}

Type 3 von Willebrand's disease (VWD) is a rare autosomal recessively inherited bleeding diathesis with an estimated prevalence of $1-5$ per million, characterized by complete or almost complete deficiency of von Willebrand factor (VWF) [1]. As VWF is the carrier for the Factor VIII (FVIII) molecule, FVIII:C levels are also generally $<10 \%$ in Type 3 disease $[2,3]$.

Correspondence: Suman L. Sood, MD, Division of HematologyOncology, University of Michigan, C351A Med Inn Building, Ann Arbor, MI 48109.

Tel.: +734.615.2681; fax: +734.615.2109;

e-mail:sumisood@med.umich.edu

${ }^{1}$ A list of the contributing Hemophilia Treatment Center Network (HTCN) Study Investigators appears in the Supporting Information.

Accepted after revision 15 January 2013
As with other forms of VWD, most patients present with mucocutaneous bleeding [4]. However, unlike Type 1 or 2 VWD, joint bleeding has been reported as a prominent symptom (rates 37-45\%) [4-7]. In 1926, Erik von Willebrand first described a traumatic ankle bleed in Hjördis, the original Aland Island proband with Type 3 VWD who bled to death from menorrhagia [8].

A survey of Type 3 VWD patients in U.S. Hemophilia Treatment Centers (HTC) found a joint bleeding rate of $40.9 \%$ and evidence of morbidity including $28.2 \%$ target joints, and $5.6 \%$ invasive joint procedures [6]. Recurrent haemarthroses lead to arthropathy, characterized by pain, joint degeneration and loss of range of motion (ROM). Radiographic findings in chronic arthropathy are similar to haemophilia [9]. ROM loss is higher in Type 3 VWD compared with Type 1 or 2, with ankles and hips the most predominantly affected joints $[10,11]$.

In 1997, the Centers for Disease Control (CDC) established the Universal Data Collection (UDC) study which tracked joint complications in patients with 
bleeding disorders. In a previous analysis conducted by our group using the UDC database, we found high rates of joint bleeding $(45 \%)$ in subjects with severe VWD, consistent with previous cohorts [12]. Low FVIII $(\leq 5 \%)$ and low VWF:Ag $(<1 \%)$ both positively correlated with higher rates of joint bleeding and were significantly associated with higher rates of joint ROM loss in a longitudinal model. Further clarification of the individual contributions of the FVIII and VWF:Ag to joint ROM loss, potentially modifiable variables which may influence the optimal choice and dose of replacement factor concentrate, could not be performed in this population due to concerns of collinearity.

Type 3 VWD patients have similar FVIII values to moderate haemophilia A (HA) of $1-5 \%$ [5]. Unlike VWD, HA patients have normal VWF levels. Joint bleeding, often traumatic, has long been recognized as a significant symptom in moderate HA. A crosssectional study in the UDC including 1048 subjects age $<20$ with moderate haemophilia A and B found that $37.8 \%$ had asymmetrically mobile joints, and joint ROM limitation increased with age, body mass index (BMI) and non-white race [13]. FVIII level was not independently considered a variable in that study, but higher FVIII levels have been associated with decreased longterm joint damage as assessed by radiologic Pettersson scoring in a Dutch moderate haemophilia cohort [14].

To further clarify the roles of FVIII and VWF:Ag, we established two cohorts of patients with similar FVIII levels using the UDC database. The purpose of this retrospective cohort study was to compare joint ROM loss and other bleeding symptoms in the moderate haemophilia A and Type 3 VWD populations, and to evaluate the contribution of the FVIII level to joint ROM over time in these subjects.

\section{Materials and methods}

\section{Patients and methods}

Between 1997 and 2011, the UDC was administered through the $\sim 140$ federally funded HTCs within the U.S. and its territories; participation was approved by the Institutional Review Board of each HTC and the CDC. Study subjects completed a standardized registration form upon enrolment (which occurred at any age), including demographics, diagnosis, baseline factor activity, retrospective recollection of first bleeding symptoms and joint ROM. UDC participation was offered annually; the number of new bleeds, treatment, BMI and joint ROM were among items documented. All participants gave written informed consent before enrolment.

Patients. The method of identifying VWD patients has previously been described in detail [12]. In brief, subjects were initially identified by diagnosis of 'Type 3 VWD' on the UDC registration form. HTCs were then contacted directly to obtain baseline laboratory values to validate this diagnosis. A 'severe VWD' cohort of subjects with VWF:Ag value $<10 \%$ was included in the prior study [12]. Among VWD patients with a validated diagnosis, only those with a FVIII level $\leq 5 \%$ were selected for inclusion in this analysis. This cohort was designated 'Type 3 VWD'. As a comparison group, all subjects with moderate HA, identified by diagnosis and baseline FVIII activity level between $1 \%$ and $5 \%$, were selected.

Inclusion/exclusion criteria. Study subjects were required to have a validated diagnosis of severe VWD or moderate HA, as defined above. Subjects with insufficient information, history of inhibitor, another bleeding disorder, or BMI > 35 (because of inaccuracy in joint ROM measurement) were excluded.

Data collection. Data were included from the Registration and Annual Visit forms of subjects from 1997 to 2007. FVIII activity level was a continuous variable. Orthopaedic appliances include casts, splints, orthotics and braces. Invasive joint procedures include arthrodesis, joint replacement, synovectomy, or other. Collection of joint ROM has been described previously [12,13]. Briefly, 10 joints (bilateral shoulders, elbows, hips, knees and ankles) are passively moved to their fullest extent and ROM is recorded. Left- and right-sided joints were averaged and then each joint ROM measure was divided by its reference value from the gender-specific normal population aged 12-20 [15]. The overall joint ROM proportion was the average of the 10 joint-specific proportions, to gain a summary measure for each individual.

Statistical analysis. Univariate analyses were carried out with the student $t$-test and chi-squared test for continuous and categorical variables respectively. Factors related to joint ROM loss over time were explored with the mixed model for continuous outcome variable with repeated measurements. A backward stepwise elimination strategy was used to generate a predictive model. The marginal model was employed to investigate factors associated with episodic bleeding occurrence during follow-up. Sensitivity analyses included evaluation of Type 3 VWD subjects with FVIII $1-5 \%$ (vs. $\leq 5 \%$ ) and removal of the invasive procedure variable in univariate and multivariate analyses. All analyses were performed using Statistical Analysis Software (SAS) version 9.1 (SAS Institute, Cary, NC).

\section{Results}

\section{Study population}

One hundred subjects with Type 3 VWD and 1814 subjects with moderate HA that met our prespecified 
criteria were identified within the UDC database. Sociodemographic information at enrolment is listed in Table 1 . As expected, the Type 3 VWD group included many more women $(0.9 \%$ HA vs. $49.0 \%$ VWD). The HA population was more racially diverse. Mean FVIII levels were similar (HA $2.9 \% \pm 1.3$ vs. VWD $2.6 \% \pm 1.4, P=n s)$, as was the age of registration.

\section{Bleeding characteristics at baseline}

High percentages of patients with both moderate HA and Type 3 VWD reported a history of any bleeding at the time of registration $(97.7 \%$ vs. $99.0 \%)$. Figure 1 shows bleeding symptoms at registration (which can occur at any age). Both groups demonstrate a strong bleeding phenotype, with similar rates of intracranial haemorrhage. Moderate HA subjects reported higher rates of family history of bleeding disorder, home factor infusion and prophylaxis usage $(20.9 \%$ vs. $12.0 \%, P=0.03)$. Type 3 VWD subjects were more likely to have ever required blood products

Table 1. Sociodemographic information at enrolment.

\begin{tabular}{|c|c|c|c|}
\hline & $\begin{array}{c}\text { Moderate } \\
\text { haemophilia } \\
\text { A }(n=1814)\end{array}$ & $\begin{array}{l}\text { VWD Type } 3 \\
(n=100)\end{array}$ & $P$ value \\
\hline \multicolumn{4}{|l|}{ Race } \\
\hline White & $1200(66.2)$ & $84(84.0)$ & 0.0002 \\
\hline African-American & $216(11.9)$ & $4(4.0)$ & \\
\hline Hispanic & $268(14.8)$ & $2(2.0)$ & \\
\hline Other & $130(7.2)$ & $10(10.0)$ & \\
\hline \multicolumn{4}{|l|}{ Gender } \\
\hline Female & $17(0.9)$ & $49(49.0)$ & $<0.0001$ \\
\hline Male & $1797(99.1)$ & $51(51.0)$ & \\
\hline $\begin{array}{l}\text { Factor VIII activity } \\
\text { level* (mean, SD) }\end{array}$ & $2.9(1.3)$ & $2.6(1.4)$ & 0.12 \\
\hline \multicolumn{4}{|l|}{ Age (years) } \\
\hline Registration (mean, SD) & $19.1(15.9)$ & $21.9(17.0)$ & 0.09 \\
\hline Range & $(2-69)$ & $(3-69)$ & \\
\hline First bleed (mean, SD) & $2.5(4.7)$ & $2.0(5.6)$ & 0.45 \\
\hline
\end{tabular}

or factor overall, and retrospectively report mucosal bleeding or bleeding after an intramuscular (IM) injection as the first site of bleeding (Fig. 2). In comparison, moderate HA subjects experienced higher rates of initial intracranial and extracranial bleeding. There was a non-significant trend towards a higher number of initial joint bleeds in the HA population $(11.1 \%$ vs. $6 \%, P=0.11)$. Employment status was similar at registration (HA vs. VWD, $25.2 \%$ vs. $25.0 \%$ reported a full or part-time job, $P=0.97)$, as was use of a cane $(19.7 \%$ vs. $17 \%, P=0.45)$ or wheelchair $(4.9 \%$ vs. $5 \%, P=0.86)$. Few patients reported a history of joint infection $(0.66 \% \mathrm{HA}$ vs. $0 \% \mathrm{VWD})$ at registration.

\section{Bleeding characteristics during follow-up}

Data from annual forms were available for a mean of 3.4 years (range $0-9.44$ ). The mean follow-up time was 3.2 years for HA and 3.9 years for VWD $(P=0.001)$. During this period, the proportion of visits in which the patient reported at least one bleed was higher in the moderate HA vs. Type 3 VWD population for joint $(46 \%$ vs. $34 \%, P<0.0001)$ and muscle bleeding $(27 \%$ vs. $16 \%, P<0.0001)$. Other bleeds, including mucosal, were higher in the Type 3 VWD vs. HA group $(49 \%$ vs. $32 \%, P<0.0001)$. More VWD than HA patients reported at least one invasive joint procedure at a follow-up visit $(6 \%$ vs. $4 \%, P=0.05)$, with slightly more synovectomy, arthrodesis and other. Distribution of joint replacement and orthopaedic appliance usage was similar (Fig. 3).

\section{Risk factors for joint range of motion loss over time}

Longitudinal multivariate analysis showed no difference in joint ROM loss over time between individuals with VWD and moderate HA when controlled for

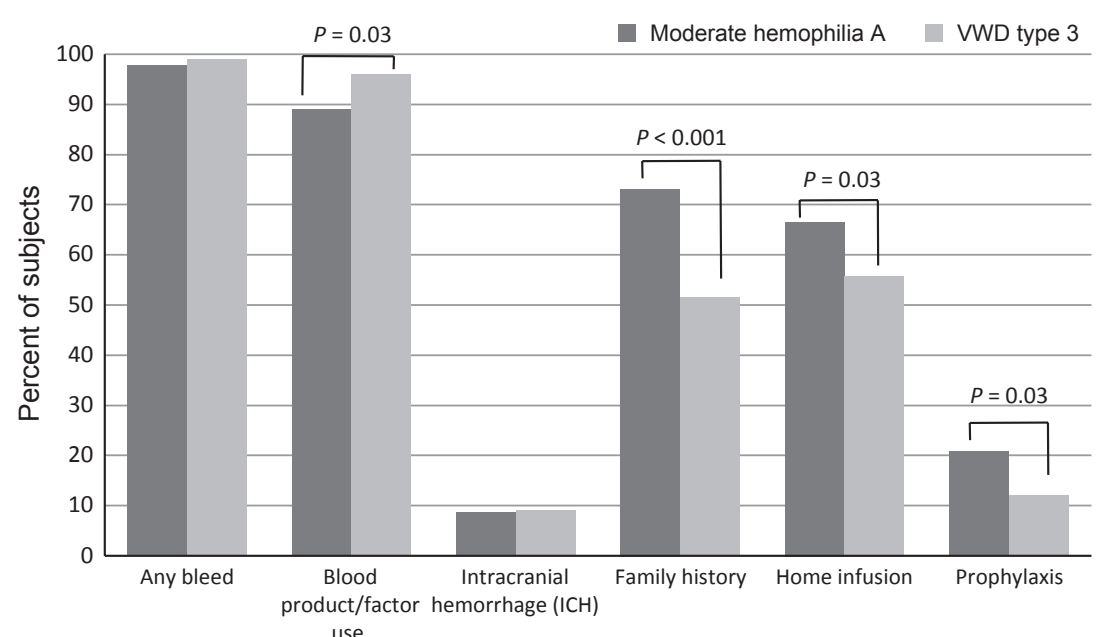

use 


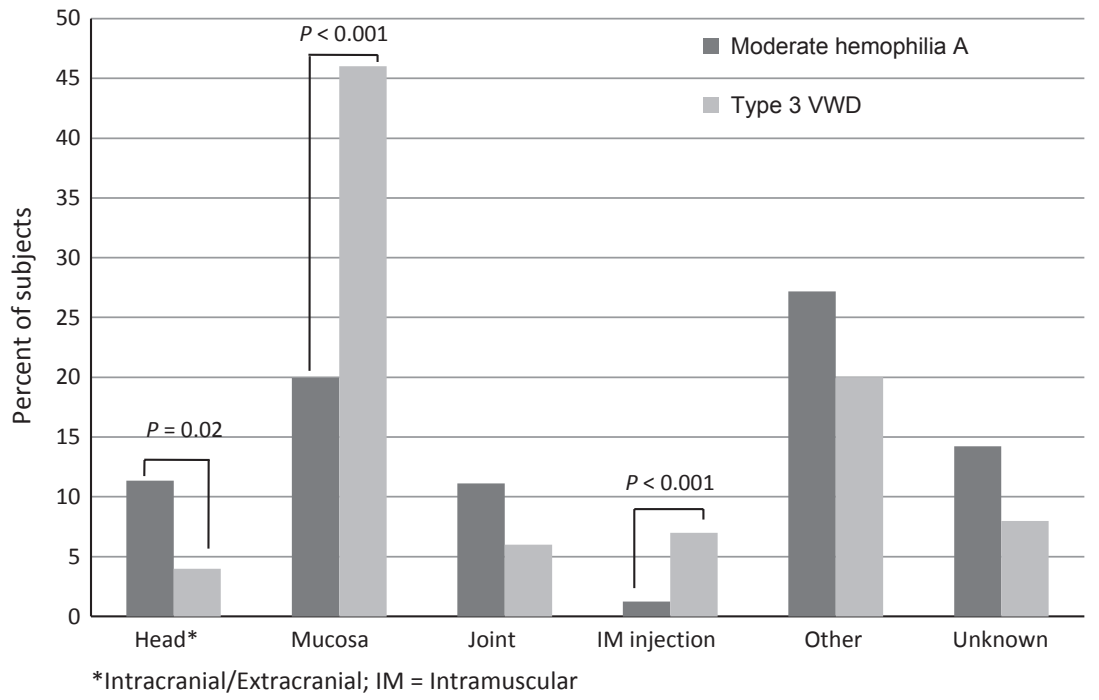

Fig. 2. Site of first bleed.

age, race, gender, FVIII level, BMI, use of prophylaxis and history of invasive joint procedures or orthopaedic appliances. Higher FVIII level was associated with preserved joint ROM $(P<0.001)$. Lower FVIII level correlated with a higher rate of joint [OR $(95 \%$ CI), $0.77(0.73-0.82), P<0.001]$ and muscle [0.84 (0.79-0.90), $P<0.001]$, but not mucosal bleeding [0.96, (0.91-1.01), $P=0.10]$.

There was no significant difference in joint ROM loss between the groups when upper and lower extremities were evaluated separately or when ankles, a problematic joint in VWD, were analysed alone. Subset analysis in subjects age $<20$, comprising $68 \%$ and $54 \%$ of all HA and VWD subject visits, respectively, again found no difference in joint ROM loss over time in the Type 3 VWD vs. moderate HA population (results not shown). As a sensitivity analysis, Type 3 VWD subjects with FVIII 1-5\% (334 visits) vs. $\leq 5 \%$ (390 visits), were also evaluated, which did not change the direction of estimated parameters or the significance of any included variables. Similarly, analysis with removal of the invasive procedure variable did not change the model.

\section{Discussion and conclusion}

We utilized a large U.S. database of patients with bleeding disorders to compare joint dysfunction in patients with Type 3 VWD and moderate HA. FVIII levels, pattern of bleeding symptoms at registration

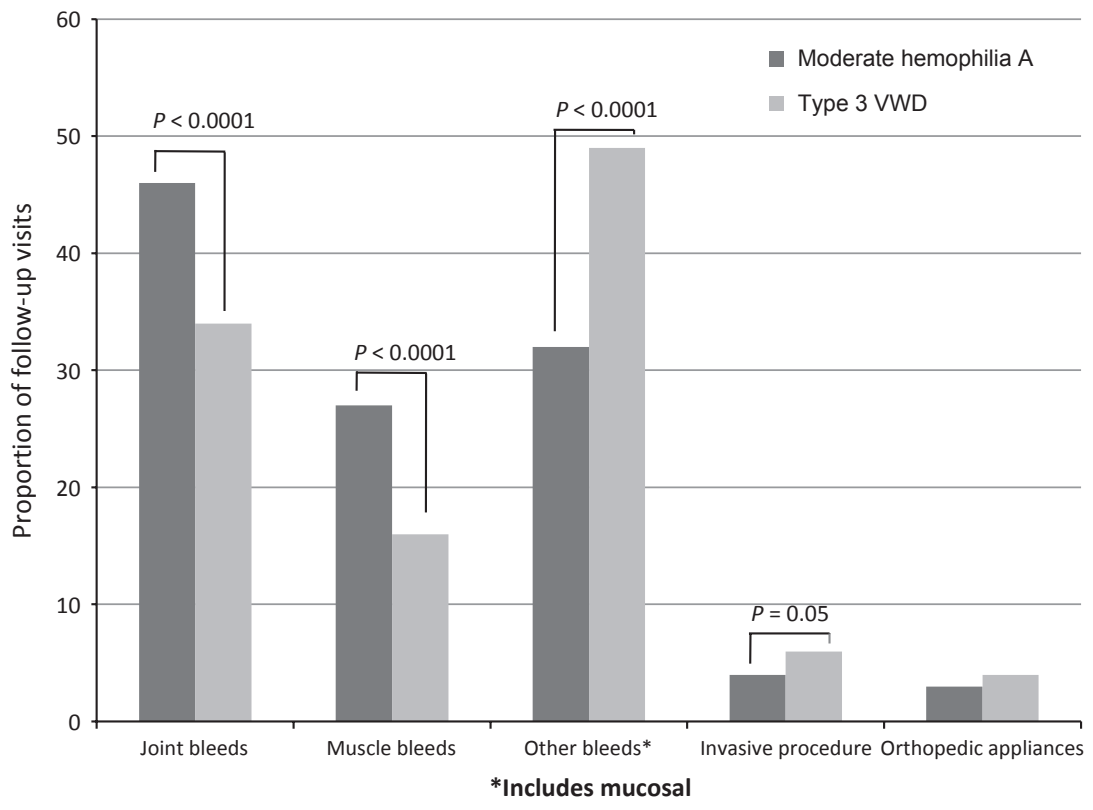

Fig. 3. Bleeding events during follow-up: proportion of visits with at least one reported bleed. 
and age at first bleed and at registration were very similar in the groups, supporting their comparability.

In our analysis, we found no difference in joint function limitation, as defined by ROM loss, in the Type 3 VWD and moderate HA populations over time. The diagnosis of VWD or HA remained nonsignificant in both the full and reduced longitudinal multivariate models, as well as in subset analyses. There was a trend towards an increased number of haemarthroses as the initial bleed site and a significant increase in visits with a reported joint bleed during the follow-up period in the HA patients.

Joint bleeding is characteristic of haemophilia, whereas patients with VWD more typically experience platelet-mediated mucosal bleeding. Joints may be particularly prone to bleed in FVIII deficiency due to the critical role of the coagulation system in the synovium: cryptic tissue factor expression allows only limited factor VIIa binding leading to inadequate thrombin burst in the absence of FVIII, compounded by high amounts of tissue factor pathway inhibitor expression [16,17]. On the other hand, once bleeding has occurred, the cycle of synovial inflammation, hypertrophy and cartilage and bone destruction begins, leading to recurrent haemarthroses and joint ROM limitations over time [18].

Factor VIII was included in the analysis as a continuous variable, and increasing levels were associated with joint function preservation in both groups. Lower FVIII also correlated with increased joint and muscle, but not mucosal, bleeding in both groups. In a previous study in severe VWD (VWF:Ag $<10 \%$ ), we found that both low FVIII $(\leq 5 \%)$ and low VWF:Ag $(<1 \%)$ levels were associated with higher rates of joint bleeding and ROM loss over time [12]. Lower FVIII levels have been previously shown to correlate with increased joint damage in moderate HA patients as well [14]. Given that VWD and HA patients have very similar FVIII levels but different VWF levels, the FVIII level appears to be the more important contributor to joint function over time. It is thus possible that the VWF protein may function only in its secondary role in the synovium, as a carrier for FVIII.

Lak et al. compared 385 Iranian subjects with Type 3 VWD to 100 subjects with severe HA [5]. They found that haemarthrosis was common in VWD, but less than severe HA (37\% vs. $86 \%, P<0.0001)$. The authors speculated that the higher FVIII levels in the VWD cohort (1-9\%, median 4\%) may account for some of these differences. In contrast, we compared Type 3 VWD subjects with FVIII levels $\leq 5 \%$ to moderate HA (FVIII levels $1-5 \%$ ), to control for this variable. Our finding that rates of ROM loss were similar supports the hypothesis of the primacy of the FVIII level.

Although joint ROM loss was similar, some clear differences between the VWD Type 3 and moderate
HA groups were identified in our analysis. Patients with moderate HA were more likely to use home infusion as well as prophylaxis, although a higher percentage of Type 3 VWD patients reported any use of blood products and factor overall. This may reflect increased physician experience and comfort with prophylaxis in HA, particularly with availability of recombinant FVIII products, as well as difficulty in obtaining insurance coverage for prophylaxis in VWD.

As expected, mucosal bleeding was much more common as a first site of bleeding in VWD. Bleeding after IM injection was also higher; perhaps less reported family history of bleeding disorder may have led to inadequate precautions with this procedure. Initial intracranial and extracranial bleeds were higher in moderate HA. During follow-up, Type 3 VWD subjects continued to report high rates of mucosal bleeding. Significantly more moderate HA patients had a visit in which they reported at least one joint or muscle bleed. Interestingly, slightly more invasive procedures were reported by VWD patients, but placement of orthopaedic appliances was similar. In light of the equivalent joint ROM limitation, these findings suggest that VWD patients may not be adequately trained to recognize joint bleeds, and thus may underreport them.

The two groups differed substantially in terms of gender, with significantly more women in the Type 3 VWD group. The 17 moderate HA women were presumably symptomatic carriers with high degrees of lyonization. Longitudinal joint ROM regression analysis was consistent with our previous finding that male gender is significantly negatively associated with joint ROM in severe VWD [12], and a study of joint ROM in normal subjects, in which females have higher joint flexibility than males at all age groups in nearly all joints [15].

Strengths of our analysis include the relatively large number of subjects with HA and Type 3 VWD, a rare bleeding disorder and the ability to follow joint ROM in individuals over time. As a sensitivity analysis, Type 3 VWD subjects with FVIII $1-5 \%$ (vs. $\leq 5 \%$ ) were evaluated, which did not change the outcomes. The U.S. population is heterogeneous and likely includes patients with a variety of genetic mutations, which helps improve the generalizability of our findings [19]. An important limitation is that the UDC had traditionally been oriented towards haemophilia although it includes all inherited bleeding disorders. As such, although we were able to distinguish mucosal bleeding symptoms at registration, mucosal bleeding during follow-up was in the 'other' category (which we presume was predominantly mucosal). The small absolute sample size of female subjects, and of patients with Type 3 VWD, limited more detailed statistical analysis. Amount of factor used, another potentially confounding variable, could not be analysed as quantitative data were not included in the UDC. Slightly more 
patients with VWD had an invasive procedure. This variable was controlled for in multivariate analysis, and sensitivity analysis did not find a difference in ROM difference between VWD and HA when it was excluded. We had insufficient power to further analyse the influence of invasive procedure types on ROM due to large variation in procedure types and joints. Finally, our definition of 'Type 3 VWD' was constrained by baseline local labs. Local laboratories used different methods and reported different lower levels of sensitivity for VWF:Ag (i.e. $<5$ vs. $<1 \mathrm{IU} \mathrm{dL}^{-1}$ ). Of those patients with a discrete or undetectable VWF:Ag level $(n=63 / 100)$, the mean (SD) VWF:Ag level was $1.4 \mathrm{IU} \mathrm{dL}^{-1}(2.5)$, consistent with current guidelines [2]. As VWF:Ag is not routinely measured in HA patients, it could not be included as an independent variable in the study.

Our finding of significant joint function limitation in both the Type 3 VWD and moderate HA populations suggests a potential role for increased prophylaxis. Whereas prophylaxis in severe haemophilia is extensively used, data regarding prophylaxis in severe VWD patients remain scant. A 2005 census of 305 Type 3 VWD patients in Europe and North America found that of the 99 patients who started prophylaxis in the prior 12 months, $41.4 \%$ were for joint-related issues [20]. In our study, relatively few patients with Type 3 VWD used prophylaxis during the study period $(12.0 \%)$.

Limited data thus far indicate that prophylaxis may benefit patients with VWD including secondary prophylaxis in several small European cohorts [20-25]. Further information will be forthcoming from the von Willebrand's disease Prophylaxis Network (vWD PN), which includes both a registry tracking use of prophylaxis, and the prospective VWD International Prophylaxis Study using a VWF-containing concentrate
1-3 times per week [26]. Results from the study thus far demonstrate significantly lower annualized rates of joint bleeding with prophylaxis in 59 individuals, including 34 (57.6\%) with Type 3 VWD [27].

In summary, we found that subjects with Type 3 VWD and moderate HA develop similar rates of joint function limitation over time. An important potentially modifiable variable identified was the FVIII level, suggesting that a low VWF:Ag may not contribute any additional risk to joint function limitation in the Type 3 VWD population. Studies are needed to further define risk factors in both the Type 3 VWD and moderate HA populations, and identify a subgroup that would benefit from risk modification and/or prophylaxis to avoid the development of disabling arthropathy.

\section{Acknowledgements}

This work was supported by NHLBI K12HL087064 (to SLS) and T32HL07971-08 (to AC), HTRS Mentored Research Award (to ADM), and by Cooperative Agreement \#U27/CCU318053 from the CDC (to BAK).

\section{Author contributions}

BAK conceived the original study question. SLS, AC, ADM and BAK designed the study, and validated the study population. CW performed primary data analysis, with assistance from JMS, and input from all authors. All authors had access to data. SLS wrote the manuscript, with substantial input and modification from all authors.

\section{Disclosures}

Some of the authors have received funding from manufacturers of factor products used to treat HA and VWD. CSL Behring (consultancy: BK, research studies: BK, SLS), Baxter Healthcare (consultancy and research studies: BK, AC), Bayer Corporation (research studies: AC), Biogen-Idec (consultancy and research studies: BK).

\section{References}

1 Sadler JE, Budde U, Eikenboom JC et al. Update on the pathophysiology and classification of von Willebrand disease: a report of the subcommittee on von Willebrand Factor. J Thromb Haemost 2006; 4: 2103-14.

2 Nichols WL, Hultin MB, James AH et al. von Willebrand disease (VWD): evidencebased diagnosis and management guidelines, the National Heart, Lung, and Blood Institute (NHLBI) expert panel report (USA). Haemophilia 2008; 14: 171-232.

3 Weiss HJ, Sussman II, Hoyer LW. Stabilization of factor VIII in plasma by the von Willebrand factor. Studies on posttransfusion and dissociated factor VIII and in patients with von Willebrand's disease. J Clin Invest 1977; 60: 390-404.

4 Federici AB. Diagnosis of inherited von Willebrand disease: a clinical perspective. Semin Thromb Hemost 2006; 32: 555-65.
5 Lak M, Peyvandi F, Mannucci PM. Clinical manifestations and complications of childbirth and replacement therapy in 385 Iranian patients with type 3 von Willebrand disease. Br J Haematol 2000; 111: 1236-9.

6 Sumner M, Williams J. Type 3 von Willebrand disease: assessment of complications and approaches to treatment - results of a patient and Hemophilia Treatment Center Survey in the United States. Haemophilia 2004; 10: 360-6.

7 Ahlberg A, Silwer J. Arthropathy in von Willebrand's disease. Acta Orthop Scand 1970; 41: 539-44.

8 Von Willebrand EA. Hereditary pseudohaemophilia. Haemophilia 1999; 5: 223-31 discussion 2.

9 Sankarankutty M, Evans DI. Chronic arthropathy in von Willebrand's disease. Clin Lab Haematol 1983; 5: 149-56.

10 Abshire TC. Prophylaxis and von Willebrand's disease (vWD). Thromb Res 2006; 118(Suppl 1): S3-7.
11 Soucie JM, Forsyth A, Watson C, Wang C, U.D.C. Investigators. Joint Range of Motion (ROM) measurements in patients with von Willebrand disease compared with normal subjects. J Thromb Heamost 2007; 5(Suppl. 2): P-W-188.

12 Metjian AD, Wang C, Sood SL et al. Bleeding symptoms and laboratory correlation in patients with severe von Willebrand disease. Haemophilia 2009; 15: 918-25.

13 Soucie JM, Cianfrini C, Janco RL et al. Joint range-of-motion limitations among young males with hemophilia: prevalence and risk factors. Blood 2004; 103: 2467-73.

14 Fischer K, van der Bom JG, Mauser-Bunschoten EP et al. Endogenous clotting factor activity and long-term outcome in patients with moderate haemophilia. Thromb Haemost 2000; 84: 977-80.

15 Soucie JM, Wang C, Forsyth A et al. Range of motion measurements: reference values and a database for comparison studies. Haemophilia 2011; 17: 500-7. 
16 Brinkmann T, Kahnert H, Prohaska W, Nordfang O, Kleesiek K. Synthesis of tissue factor pathway inhibitor in human synovial cells and chondrocytes makes joints the predilected site of bleeding in haemophiliacs. Eur J Clin Chem Clin Biochem 1994; 32: 313-7.

17 Berckmans RJ, Nieuwland R, Tak PP et al. Cell-derived microparticles in synovial fluid from inflamed arthritic joints support coagulation exclusively via a factor VII-dependent mechanism. Arthritis Rheum 2002; 46: 2857-66.

18 Valentino LA, Hakobyan N, Enockson C. Blood-induced joint disease: the confluence of dysregulated oncogenes, inflammatory signals, and angiogenic cues. Semin Hematol 2008; 45: S50-7.

19 Lillicrap D. Genotype/phenotype association in von Willebrand disease: is the glass half full or empty?. J Thromb Haemost 2009; 7(Suppl 1): 65-70.
20 Berntorp E. Prophylaxis and treatment of bleeding complications in von Willebrand disease type 3. Semin Thromb Hemost 2006; 32: 621-5.

21 Berntorp E, Petrini P. Long-term prophylaxis in von Willebrand disease. Blood Coagul Fibrinolysis 2005; 16(Suppl 1): S23-6.

22 Berntorp E. Prophylaxis in von Willebrand disease. Haemophilia 2008; 14(Suppl 5): 47-53.

23 Federici AB. Prophylaxis of bleeding episodes in patients with von Willebrand's disease. Blood Transfus 2008; 6(Suppl 2): s26-32.

24 Federici AB, Gianniello F, Canciani MT, Mannucci PM Secondary long-term prophylaxis in severe patients with von Willebrand' disease: an Italian cohort study. ASH Annual Meeting Abstracts 2005; 106: 1782.

25 Halimeh S, Krumpel A, Rott $\mathrm{H}$ et al. Long-term secondary prophylaxis in children, adolescents and young adults with von Willebrand disease. Results of cohort study. Thromb Haemost 2011; 105: 597-604.

26 Berntorp E, Abshire T. The von Willebrand disease prophylaxis network (vWD PN): exploring a treatment concept. Thromb Res 2006; 118(Suppl 1): S19-22.

27 Abshire TC, Federici AB, Alvarez MT et al. Prophylaxis in severe forms of von Willebrand's disease: results from the von Willebrand Disease Prophylaxis Network (VWD PN). Haemophilia 2013; 19: 76-81.

\section{Supporting Information}

Additional Supporting Information may be found in the online version of this article:

Appendix S1. List of contributing investigators. 